\title{
OPTIMAL TIME TO EXCHANGE TWO BASKETS
}

\author{
KATSUMASA NISHIDE, ${ }^{*}$ Yokohama National University \\ L. C. G. ROGERS, ${ }^{* *}$ University of Cambridge
}

\begin{abstract}
In this paper we present simple extensions of earlier works on the optimal time to exchange one basket of $\log$ Brownian assets for another. A superset and subset of the optimal stopping region in the case where both baskets consist of multiple assets are obtained. It is also shown that a conjecture of $\mathrm{Hu}$ and Øksendal (1998) is false except in the trivial case where all the assets in a basket are the same processes.

Keywords: Log Brownian motion; optimal stopping time; continuation region; convex hull

2010 Mathematics Subject Classification: Primary 60G40
\end{abstract}

Secondary 62L15; 93E20

\section{Introduction}

In the real options literature, optimal investment timing is often examined under the assumption that revenue and/or cost processes follow log Brownian motions. As mentioned in [3], this specification can be justified when the processes represent their present values. Moreover, revenues and costs may consist of multiple sources in practice, implying that it is important to analyze the case in which both revenues and costs follow additive log Brownian motions. The problem to be studied in this paper is the optimal stopping time

$$
\sup _{\tau} \mathrm{E}\left[\mathrm{e}^{-\rho \tau}\left(X_{\tau}^{1}+\cdots+X_{\tau}^{n}-X_{\tau}^{n+1}-\cdots-X_{\tau}^{n+m}\right)\right],
$$

where $\rho$ is the discount rate and $\left(X^{1}, \ldots, X^{n+m}\right)$ are log Brownian motions representing the net present values of revenues and costs. Because of mathematical intractability, only a few studies investigate problem (1.1) analytically. The possibility that $\tau=\infty$ is allowed, and corresponds to the situation where the investor never exercises his option to invest in the project. Accordingly, we make the convention that

$$
\mathrm{e}^{-\rho \tau}\left(X_{\tau}^{1}+\cdots+X_{\tau}^{n}-X_{\tau}^{n+1}-\cdots-X_{\tau}^{n+m}\right)=0 \quad \text { if } \tau=\infty .
$$

Since the agent may postpone his moment of investment for ever if need be, it is clear that he would never invest at any time when the value of investing was negative, and the value to him of the option is automatically nonnegative.

The first study on optimal investment timing of this type was by McDonald and Siegel [3]. They considered the $n=m=1$ case and showed that the optimal stopping time $\tau^{*}$ takes the form

$$
\tau^{*}=\inf \left\{t \geq 0: X_{t}^{1} \geq \frac{X_{t}^{2}}{z^{*}}\right\},
$$

Received 3 June 2010; revision received 9 November 2010.

* Postal address: International Graduate School of Social Sciences, Yokohama National University, Yokohama 240-8501, Japan. Email address: knishide@ynu.ac.jp

** Postal address: Statistical Laboratory, University of Cambridge, Cambridge CB3 0WB, UK. 
where the constant $z^{*}$ is determined by the values of the drifts, volatilities, and correlation coefficient of the two processes.

Olsen and Stensland [4] considered the problem in the case $n=1$. They proved that the optimal stopping region of (1.1) contains the cone

$$
\left\{x \in \mathbb{R}_{+}^{m+1}: x_{1} \geq \sum_{k=2}^{m+1} \frac{x_{k}}{z_{1 k}^{*}}\right\},
$$

where $z_{1 k}^{*}$ denotes the barriers for the two-dimensional case of $X^{1}$ and $X^{k}$. They obtained a similar result for the $m=1$ case.

$\mathrm{Hu}$ and $\varnothing \mathrm{ksendal}$ [2] studied the problems in these two papers and provided sufficient conditions for the stopping region to be equal to (1.2) in the problem of Olsen and Stensland [4]. We note that $\mathrm{Hu}$ and $\varnothing \mathrm{ksendal}$ insisted that the stopping time $\tau$ be finite. This assumption corresponds to a situation where the investor is compelled to invest at some time, and is different from our assumption; McDonald and Siegel [3] in effect made the same assumption as we do, though this slips in implicitly in their account. In the case where the discount rate $\rho$ is greater than all the growth rates of the $X^{i}$, then there is no difference in the solution.

In this paper we study the optimal stopping problem for the general case (1.1) analytically. Our first results, in Section 2, provide bounds on the stopping set. On the one hand, we prove that the stopping region for (1.1) contains a cone represented as the convex hull of a union of simpler sets, and as we shall see, this generalizes the result of Olsen and Stensland [4]. On the other hand, we obtain a superset that contains the stopping region, extending the result of $\mathrm{Hu}$ and Øksendal [2], proved only for the $n=1$ case.

Our second theme, presented in Section 3, is to prove that the stopping region is in general quite a complicated shape, and the simple cones identified in Section 2 are generally strict sub/supersets. We prove that in the case in which $n=1$ and $m=2$, studied by Olsen and Stensland [4] and $\mathrm{Hu}$ and Øksendal [2], the convex cone (1.2) identified as a subset of the stopping region is strictly contained in the stopping region unless the problem is degenerate. More precisely, the sufficient conditions for the equivalence that $\mathrm{Hu}$ and $\emptyset \mathrm{ksendal}$ [2] presented hold only in a degenerate case where the volatility matrix of $\log$ Brownian motions is singular. The result of Section 3 has been independently proved by Christensen and Irle [1], using quite different techniques.

Finally, concluding remarks are given in Section 4.

\section{Problem formulation and basic results}

We first present the simplest form of the problem, which is a one-dimensional problem solvable in closed form. Though very simple, this is the key result (due to McDonald and Siegel [3]); all subsequent results in this paper use this by suitable comparison with the basic one-dimensional problem.

The problem is to find the optimum

$$
v(z) \equiv \sup _{\tau} \mathrm{E}^{z}\left[\mathrm{e}^{-\rho \tau}\left(1-Z_{\tau}\right)\right]
$$

where $Z$ is a $\log$ Brownian motion

$$
\mathrm{d} Z_{t}=Z_{t}\left(\sigma \mathrm{d} W_{t}+\mu \mathrm{d} t\right), \quad Z_{0}=z>0,
$$

$\rho>0$ is fixed, and $\tau \geq 0$ is a stopping time.

The solution is easy to determine. 
Proposition 2.1. The value function $v$ has the form

$$
v(z)= \begin{cases}1-z, & z \leq z^{*}, \\ \left(1-z^{*}\right)\left(\frac{z}{z^{*}}\right)^{-\alpha}, & z \geq z^{*}\end{cases}
$$

where $-\alpha<0$ is the negative root of the quadratic

$$
Q(t) \equiv \frac{1}{2} \sigma^{2} t(t-1)+\mu t-\rho
$$

and

$$
z^{*}=\frac{\alpha}{1+\alpha}
$$

The optimal stopping time is to take

$$
\tau=\inf \left\{t: Z_{t} \leq z^{*}\right\}
$$

Proof. Let $\mathcal{L}$ be the operator given by

$$
\mathcal{L}=\frac{\sigma^{2}}{2} z^{2} \frac{\partial^{2}}{\partial z^{2}}+\mu z \frac{\partial}{\partial z} .
$$

The following properties of the function $v$ defined by (2.1) are easy to verify:

(P1) $v(z) \geq 1-z$ for all $z>0$;

(P2) $v$ is $C^{1}$;

(P3) $(\mathcal{L}-\rho) v(z) \leq 0$ for all $z>0$ with equality for $z>z^{*}$.

Hence, by Itô's formula,

$$
\mathrm{e}^{-\rho t} v\left(Z_{t}\right)=v\left(Z_{0}\right)+\int_{0}^{t} \mathrm{e}^{-\rho s} v^{\prime}\left(Z_{s}\right) \sigma Z_{s} \mathrm{~d} W_{s}+\int_{0}^{t} \mathrm{e}^{-\rho s}(\mathcal{L}-\rho) v\left(Z_{s}\right) \mathrm{d} s .
$$

(The $C^{1}$ property is used here; if there was a discontinuity of the gradient of $v$ at $z^{*}$, there would also be a term involving the local time of $Z$ at $z^{*}$.) Since the payoff function is bounded, the local martingale term on the right-hand side of (2.2) is also bounded (all the other terms in (2.2) are clearly bounded), implying that it is in fact a martingale. Hence, by the optional sampling theorem we have, for any stopping time $T$,

$$
\begin{aligned}
\mathrm{E}\left[\mathrm{e}^{-\rho T}\left(1-Z_{T}\right)\right] & \leq \mathrm{E}\left[\mathrm{e}^{-\rho T} v\left(Z_{T}\right)\right] \\
& =v\left(Z_{0}\right)+\mathrm{E} \int_{0}^{T} \mathrm{e}^{-\rho s}(\mathcal{L}-\rho) v\left(Z_{s}\right) \mathrm{d} s \\
& \leq v\left(Z_{0}\right),
\end{aligned}
$$

using properties (P1) and (P3). Thus, $v\left(Z_{0}\right)$ is an upper bound for the value starting from $Z_{0}$. This bound is seen to be achieved when $T=\tau$ because (a) $v\left(Z_{\tau}\right)=1-Z_{\tau}$ and (b) up until $\tau$, $(\mathcal{L}-\rho) v\left(Z_{s}\right)=0$. 
Remark 2.1. Theorem 3.2 of $\mathrm{Hu}$ and $\emptyset \mathrm{ksendal}$ [2] states only that (2.1) holds when $\mu \leq \sigma^{2} / 2$, and it is unclear whether the equation holds when $\mu>\sigma^{2} / 2$. (In this setting, the condition $\mu \leq \sigma^{2} / 2$ is equivalent to $\mathrm{P}\{\tau<\infty\}=1$. The discrepancy is due to the assumption in [2] that the stopping time must be finite.) Proposition 2.1 ensures that the value function is given by (2.1) under any parameter values. Note also that the value function $v$ constructed is $C^{1}$, but fails to be $C^{2}$ at $z^{*}$.

To formulate the multidimensional analogue of this problem, we suppose that $W$ is a standard Brownian motion in $\mathbb{R}^{N}$, and that $\log$ Brownian motions $X_{t}^{i}, i=1, \ldots, n+m$, are defined as the solutions to the equations

$$
\mathrm{d} X_{t}^{i}=X_{t}^{i}\left(\sigma_{i} \cdot \mathrm{d} W_{t}+\mu_{i} \mathrm{~d} t\right), \quad X_{0}^{i}=x_{i} .
$$

Here, the $\sigma_{i}$ are fixed vectors in $\mathbb{R}^{N}$ and the $\mu_{i}$ are fixed scalars.

The multidimensional problem is to find the value function

$$
V(x) \equiv \sup _{\tau} \mathrm{E}^{x}\left[\mathrm{e}^{-\rho \tau}\left(\sum_{j=1}^{n} X_{\tau}^{j}-\sum_{k=n+1}^{n+m} X_{\tau}^{k}\right)\right]
$$

and the optimal stopping region in $\mathbb{R}^{n+m}$. We make the notational convention that an index $j$ is assumed to be in $\{1, \ldots, n\}$, and an index $k$ is assumed to be in $\{n+1, \ldots, n+m\}$ unless stated explicitly to the contrary. We also make the notational convention that $\sum_{j} \equiv \sum_{j=1}^{n}$ and $\sum_{k} \equiv \sum_{k=n+1}^{n+m}$. We shall assume that

$$
\rho>\mu_{j} \text { for all } j=1, \ldots, n,
$$

otherwise the problem may fail to be well posed.

This problem will not in general be explicitly solvable except in the trivial case where all the log Brownian motions are the same, but we will be able to find out some useful information about the stopping region. We are however able to find the solution explicitly in the case where $n=m=1$ by reducing the problem to the one-dimensional problem solved in Proposition 2.1. To see how this works, consider

$$
v_{j k}(x) \equiv v_{j k}\left(x_{j}, x_{k}\right) \equiv \sup _{\tau} \mathrm{E}\left[\mathrm{e}^{-\rho \tau}\left(X_{\tau}^{j}-X_{\tau}^{k}\right) \mid X_{0}=x\right]
$$

By the change of probability measures, we have

$$
v_{j k}(x)=\sup _{\tau} x_{j} \tilde{\mathrm{E}}\left[\mathrm{e}^{-\left(\rho-\mu_{j}\right) \tau}\left(1-Z_{\tau}\right) \mid X_{0}=x\right],
$$

where we have written

$$
Z_{t} \equiv \frac{X_{t}^{k}}{X_{t}^{j}}
$$

a $\log$ Brownian motion, and we write $\tilde{E}$ for expectation with respect to the probability $\tilde{\mathrm{P}}$ defined by

$$
\left.\frac{\mathrm{d} \tilde{\mathrm{P}}}{\mathrm{dP}}\right|_{\mathcal{F}_{t}}=x_{j}^{-1} \mathrm{e}^{-\mu_{j} t} X_{t}^{j} .
$$


As is well known, the effect of changing the measure to $\tilde{\mathrm{P}}$ is to introduce a drift into the Brownian motion $W$ :

$$
\tilde{W}_{t} \equiv W_{t}-\sigma_{j} t
$$

is a $\tilde{\mathrm{P}}$-Brownian motion. Thus, under the probability measure $\tilde{\mathrm{P}}, Z$ is also a $\log$ Brownian motion (explicitly, $\mathrm{d} Z_{t}=Z_{t}\left\{\left(\sigma_{k}-\sigma_{j}\right) \cdot \mathrm{d} \tilde{W}_{t}+\left(\mu_{k}-\mu_{j}\right) \mathrm{d} t\right\}$ ), and the value function $v_{j k}$ has the form given by Proposition 2.1. The stopping region for this problem is the region where

$$
Z_{t} \equiv \frac{X_{t}^{k}}{X_{t}^{j}} \leq z_{j k}^{*},
$$

where the constant $z_{j k}^{*}$ is calculated analogously.

Returning to the multidimensional problem (2.4), we write

$$
g(x) \equiv \sum_{j} x_{j}-\sum_{k} x_{k}
$$

for the stopping value, and we note that $V \geq g$. As Olsen and Stensland [4] observed, for any stopping time $\tau$, the function $x \mapsto \mathrm{E}^{x} \mathrm{e}^{-\rho \tau} g\left(X_{\tau}\right)$ is clearly linear, so the value function $V$, which is the supremum of all such functions as $\tau$ varies, must be convex. This implies that the function $V-g \geq 0$ is convex, and, hence, the stopping region

$$
\delta \equiv\{x: V(x)=g(x)\}
$$

must be convex. For any $1 \leq j \leq n$ and $n<k \leq n+m$, we know from (2.7) that all points of the set

$$
\Delta_{j k} \equiv\left\{x: x_{k} \leq x_{j} z_{j k}^{*}, x_{i}=0 \text { for all } i \neq j, k\right\}
$$

are points of $\&$, so

$$
s \supseteq s_{-} \equiv \operatorname{Co}\left(\bigcup_{j, k} \Delta_{j k}\right),
$$

where $\operatorname{Co}(A)$ denotes the convex hull of $A$. In the case in which $n=1$, it is not hard to see that we recover the result of Olsen and Stensland [4]:

$$
s \supseteq\left\{x: x_{1} \geq \sum_{k} \frac{x_{k}}{z_{1 k}^{*}}\right\} .
$$

We shall now find a superset of the stopping set by a different comparison with the simple case $n=m=1$. We introduce $\log$ Brownian motions $X^{0}$ and $X^{K}$, solving the corresponding stochastic differential equation (2.3) for vectors $\sigma_{0}, \sigma_{K}$ and scalars $\mu_{0}, \mu_{K}<\rho$ yet to be specified, and with the initial conditions $X_{0}^{0}=X_{0}^{K}=1$. We shall, for concreteness, assume that $K=n+m+1$, so that we are considering a sequence $X^{0}, \ldots, X^{K}$ of $\log$ Brownian motions, the first and last of which are for comparison. The idea of this comparison is due to $\mathrm{Hu}$ and $\varnothing \mathrm{ksendal}[2]$.

Proposition 2.2. The stopping set 8 is contained in the set

$$
\varsigma_{+} \equiv\left\{x: z_{0 K}^{*} \sum_{j} \frac{x_{j}}{z_{0 j}^{*}} \geq \sum_{k} x_{k} z_{k K}^{*}\right\},
$$

whatever $\sigma_{0}, \sigma_{K}$ and $\mu_{0}, \mu_{K}<\rho$. 
Remark 2.2. Note that the set $\varsigma_{+}$depends on the dynamics of $X^{0}$ and $X^{K}$; if we allow $\sigma_{0}$, $\sigma_{K}, \mu_{0}$, and $\mu_{K}$ to vary, we find that the stopping region is contained in the convex hull of an intersection of half-spaces of the form (2.9).

Proof of Proposition 2.2. For any stopping time $\tau$ and any positive $\xi_{i}, i=1, \ldots, n+m$, we have

$$
\mathrm{Ee}^{-\rho \tau}\left(X_{\tau}^{j}-\xi_{j} X_{\tau}^{0}\right) \geq-v_{0 j}\left(\xi_{j}, x_{j}\right), \quad \mathrm{Ee}^{-\rho \tau}\left(X_{\tau}^{k}-\xi_{k} X_{\tau}^{K}\right) \leq v_{k K}\left(x_{k}, \xi_{k}\right) .
$$

Hence, for any stopping time $\tau$,

$$
\begin{aligned}
V(x) & \geq \mathrm{E}\left[\mathrm{e}^{-\rho \tau}\left(\sum_{j} X_{\tau}^{j}-\sum_{k} X_{\tau}^{k}\right)\right] \\
& \geq \mathrm{E}\left[\mathrm{e}^{-\rho \tau}\left(X_{\tau}^{0} \sum_{j} \xi_{j}-X_{\tau}^{K} \sum_{k} \xi_{k}\right)\right]-\sum_{j} v_{0 j}\left(\xi_{j}, x_{j}\right)-\sum_{k} v_{k K}\left(x_{k}, \xi_{k}\right) .
\end{aligned}
$$

Now taking the supremum over all stopping times, we obtain the inequality

$$
V(x) \geq v_{0 K}\left(\sum_{j} \xi_{j}, \sum_{k} \xi_{k}\right)-\sum_{j} v_{0 j}\left(\xi_{j}, x_{j}\right)-\sum_{k} v_{k K}\left(x_{k}, \xi_{k}\right) .
$$

Since the choices of $\xi_{i}, \sigma_{0}, \sigma_{K}, \mu_{0}$, and $\mu_{K}$ are arbitrary, we could obtain a lower bound for $V(x)$ by taking the supremum on the right-hand side of (2.10) over all these variables. However, the dependence on these variables is sufficiently complicated that it is very hard to see what may result. Nevertheless, we may exploit (2.10) by choosing the $\xi_{i}$ at the critical values, i.e.

$$
\xi_{j}=\frac{x_{j}}{z_{0 j}^{*}}, \quad \xi_{k}=x_{k} z_{k K}^{*}
$$

which ensures that

$$
\sum_{j} v_{0 j}\left(\xi_{j}, x_{j}\right)=\sum_{j}\left(\xi_{j}-x_{j}\right), \quad \sum_{k} v_{k K}\left(x_{k}, \xi_{k}\right)=\sum_{k}\left(x_{k}-\xi_{k}\right) .
$$

We will be able to deduce that $V(x)>g(x)$ if

$$
v_{0 K}\left(\sum_{j} \xi_{j}, \sum_{k} \xi_{k}\right)>\sum_{j} \xi_{j}-\sum_{k} \xi_{k}
$$

which is ensured if

$$
z_{0 K}^{*} \sum_{j} \xi_{j}=z_{0 K}^{*} \sum_{j} \frac{x_{j}}{z_{0 j}^{*}}<\sum_{k} \xi_{k}=\sum_{k} x_{k} z_{k K}^{*}
$$

If this inequality is satisfied then we know that it is optimal to continue at $x$; hence, the stopping region is contained in the set

$$
\S_{+} \equiv\left\{x: z_{0 K}^{*} \sum_{j} \frac{x_{j}}{z_{0 j}^{*}} \geq \sum_{k} x_{k} z_{k K}^{*}\right\}
$$

as claimed. 


\section{Strict inclusion of the stopping region}

$\mathrm{Hu}$ and Øksendal [2] conjectured that inclusion (2.8), found for the stopping region in the $n=1$ case, might in fact hold in wide generality. However, as we shall show next, it holds only in degenerate cases.

Proposition 3.1. Suppose that $m \geq 2$ or $n \geq 2$. When the volatility vectors are linearly independent, the convex hull is strictly included by the optimal stopping region.

Proof. To prove the proposition, we study only the situation in which $n=1$ and $m=2$. As at (2.5), we shall rephrase the problem as

$$
\sup _{\tau} \mathrm{E}^{x}\left[\mathrm{e}^{-\rho \tau}\left(X_{\tau}^{1}-X_{\tau}^{2}-X_{\tau}^{3}\right)\right]=\sup _{\tau} x_{1} \tilde{\mathrm{E}}^{x}\left[\mathrm{e}^{-\left(\rho-\mu_{1}\right) \tau}\left(1-Z_{\tau}^{2}-Z_{\tau}^{3}\right)\right]
$$

where $Z^{k} \equiv X^{k} / X^{1}$, as at (2.6). Since the $Z^{k}$ are $\log$ Brownian motions, we lose no generality in seeking the value function

$$
V(x) \equiv \sup _{\tau} \mathrm{E}^{x}\left[\mathrm{e}^{-\rho \tau}\left(1-X_{\tau}^{1}-X_{\tau}^{2}\right)\right],
$$

where $X^{1}$ and $X^{2}$ satisfy (2.3). Our proof rests on the elementary inequality valid for all $x, y>0$, and $p \equiv 1-q \in[0,1]$ :

$$
x+y=p \frac{x}{p}+q \frac{y}{q} \geq\left(\frac{x}{p}\right)^{p}\left(\frac{y}{q}\right)^{q} .
$$

This holds with equality if and only if $q x=p y$ (Young's inequality). Thus, if we define a new $\log$ Brownian motion

$$
X_{t}(p) \equiv\left(\frac{X_{t}^{1}}{p}\right)^{p}\left(\frac{X_{t}^{2}}{q}\right)^{q}
$$

for $p \in[0,1]$, the associated value function

$$
V_{p}(x) \equiv \sup _{\tau} \mathrm{E}^{x}\left[\mathrm{e}^{-\rho \tau}\left(1-X_{\tau}(p)\right)\right]
$$

is an upper bound for $V$. Calculating the value function $V_{p}$ is simply an application of Proposition 2.1; we find that it is optimal to stop whenever $\left(x_{1} / p\right)^{p}\left(x_{2} / q\right)^{q} \leq z^{*}(p)$, where

$$
z^{*}(p) \equiv \frac{\alpha(p)}{1+\alpha(p)},
$$

and $-\alpha(p)<0$ solves the quadratic

$$
\frac{1}{2} \sigma(p)^{2} t^{2}+m(p) t-\rho=0 .
$$

Here, $\sigma(p)^{2}=\left|p \sigma_{1}+q \sigma_{2}\right|^{2}$ and $m$ is the linear function

$$
m(p)=p \mu_{1}+q \mu_{2}-\frac{1}{2} p\left|\sigma_{1}\right|^{2}-\frac{1}{2} q\left|\sigma_{2}\right|^{2} .
$$

If we consider the line $L_{p} \equiv\{(\lambda p, \lambda q): \lambda \geq 0\}$ (see Figure 1) then at all points of this line where $\lambda \leq z^{*}(p)$ the optimal rule for $V_{p}$ is to stop. Thus, at all points of the line $L_{p}$ for $\lambda \leq z^{*}(p)$, we shall have $V_{p}(\lambda p, \lambda q)=1-\lambda$; what we require to prove is that the point $z^{*}(p)$ 


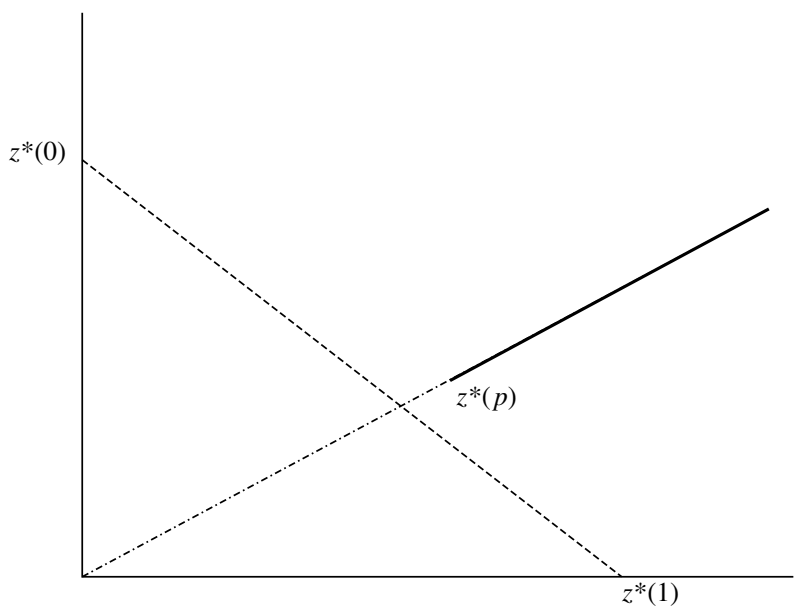

FIgURE 1: Critical region in the $\left(x_{1}, x_{2}\right)$-plane.

on this line where the rule changes lies strictly outside the triangle defined by the origin and the points $\left(0, z^{*}(0)\right)$ and $\left(z^{*}(1), 0\right)$. If this is the case, since $V_{p} \geq V$, we know that it will also be optimal to stop for $V$ all along $L_{p}$ as far as $z^{*}(p)$, and, hence, the stopping region for $V$ extends strictly outside the triangle. Routine coordinate geometry gives us that the point where $L_{p}$ reaches the edge of the triangle is where

$$
\lambda=\frac{z^{*}(0) z^{*}(1)}{p z^{*}(0)+q z^{*}(1)},
$$

which we want to be strictly smaller than $z^{*}(p)$. Equivalently stated, we require, for $0<p<1$, that

$$
\frac{1}{z^{*}(p)}<\frac{p}{z^{*}(1)}+\frac{q}{z^{*}(0)}
$$

or again, using (3.1), this is the statement that $p \mapsto 1 / \alpha(p)$ is strictly convex. It is easy to see from (3.2) that $1 / \alpha(p)$ is the positive root of the quadratic

$$
\rho t^{2}+m(p) t-\frac{1}{2} \sigma(p)^{2}=0 .
$$

This positive root is

$$
\frac{1}{\alpha(p)}=\frac{-m(p)+\sqrt{\xi(p)}}{2 \rho}
$$

where

$$
\xi(p) \equiv m(p)^{2}+2 \rho \sigma(p)^{2} \equiv a p^{2}+b p+c
$$

is quadratic in $p$. Since $m$ is linear, the convexity of $1 / \alpha(p)$ is equivalent to the convexity of $\sqrt{\xi(p)}$. Differentiating twice, after some calculations we discover that

$$
\frac{\mathrm{d}^{2}}{\mathrm{~d} p^{2}} \sqrt{\xi(p)} \geq 0 \quad \Longleftrightarrow \quad 4 a c-b^{2} \geq 0 .
$$

However, we observe that the quadratic $\xi$ is nonnegative, so cannot have two distinct real roots. Hence, $b^{2}-4 a c \leq 0$, as required.

The theorem immediately holds for general $n$ and $m$ since the stopping region is strictly contained by the convex hull on the three-dimensional plane. 


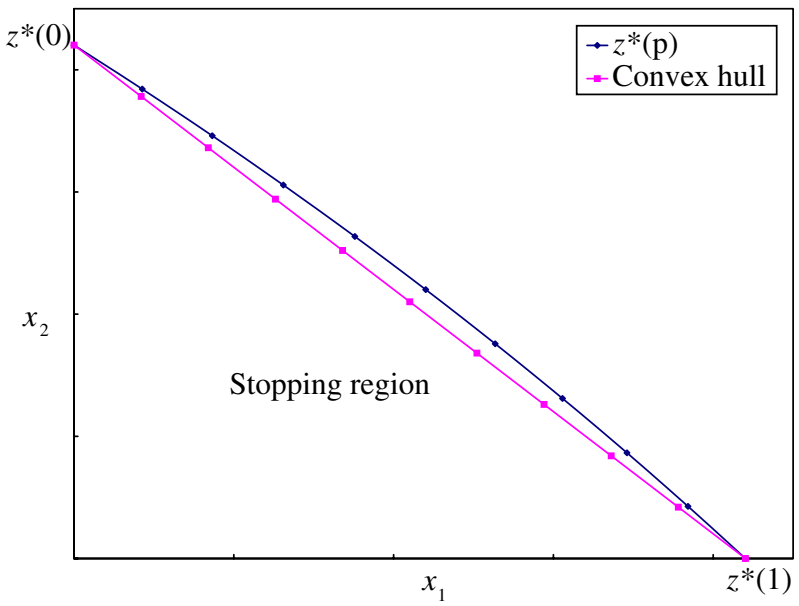

Figure 2: Convex hull and the subset obtained by $z^{*}$ in the $\left(x_{1}, x_{2}\right)$-plane.

Remark 3.1. Christensen and Irle [1] independently proved the same result. Their proof is based on a good choice of measure transform to estimate the optimal stopping value, and is an application of a general method. The proof we give here is simpler in that it requires only an elementary inequality and comparisons with the known solution to the one-dimensional problem, but is not capable of generalization.

Figure 2 presents an illustrative example of the convex hull and the subset of stopping regions described by $z^{*}(p)$. The subset induced by $\left\{z^{*}(p) ; p \in[0,1]\right\}$ is strictly greater than the convex hull. Note that the maximum subset of the optimal stopping region derived analytically in the previous studies is the convex hull of the stopping regions for the two-dimensional cases. In the proof of Proposition 3.1, we obtained a subset that is strictly greater than the convex hull. This observation helps us understand the optimal stopping region for the exchange of two baskets that contain multiple log Brownian motions.

\section{Conclusions}

In this paper we have generalized the results of Olsen and Stensland [4] and Hu and Øksendal [2], and studied the optimal stopping problem in which both positive and negative baskets are made up of linearly additive log Brownian motions.

The two main contributions of this paper are as follows. Firstly, we obtained results that included the results of Olsen and Stensland [4] and $\mathrm{Hu}$ and Øksendal [2] as special cases. That is, the stopping region for the original problem contains the convex hull of the stopping regions for all two-dimensional cases. In addition, we presented a superset that contains the stopping region in the general case where both baskets consist of multiple log Brownian motions.

Secondly, we showed that Hu and Øksendal's sufficient conditions for the equivalence of the stopping region and the convex hull only hold for a degenerate setting. More specifically, if we assume a regular volatility matrix, the optimal stopping region is strictly larger than the convex hull. Contrary to Christensen and Irle [1], our proof is based on an elementary inequality and so helps us understand how we optimally stop and exchange the two baskets. 


\section{References}

[1] Christensen, S. and Irle, A. (2011). A harmonic-function technique for the optimal stopping diffusions. To appear in Stochastics.

[2] Hu, Y. AND ØKSEndal, B. (1998). Optimal time to invest when the price processes are geometric Brownian motions. Finance Stoch. 2, 295-310.

[3] McDonald, R. And Siegel, D. (1986). The value of waiting to invest. Quart. J. Econom. 101, 707-728.

[4] Olsen, T. E. and Stensland, G. (1992). On optimal timing of investment when cost components are additive and follow geometric Brownian diffusions. J. Econom. Dynamics Control 16, 39-51. 